\title{
Vitamin B12 concentrations and clinical spectrum of appar- ently healthy elderly population
}

\author{
Darshana Makwana ${ }^{1}$ Deepak Kumar P2 , Pragathi Wadkar ${ }^{3}$
}

\author{
${ }^{1}$ Assistant Professor, Department of Medicine, B.J. Medical college, Ahmedabad, Gujarat \\ ${ }^{2}$ Deepak Kumar P, Assistant Professor, Department of Medicine, Vydehi Institute of Medical Sciences and Research Centre, \\ Bangalore, Karnataka
}

${ }^{3}$ Pragathi Wadkar, Telemedicine Medical officer, Indira Gandhi Institute of Child Health, Bangalore, Karnataka

Corresponding author: Dr Deepak Kumar P; Address- No E 1108, Parimala Sunridge apartment, Siddapura, Whitefield, Bangalore560066, Karnataka; Phone- 8238034057;

e-mail address-deepak.drdeep.kumar@gmail.com

\section{ABSTRACT}

Introduction : Prevalence of vitamin B12 deficiency is believed to increase with aging. The wide and non-specific spectrum of clinical presentations in patients poses a tough challenge to physicians suspecting vitamin B12 deficiency.

Objectives : This study was conducted to collect data on prevalence and clinical features of vitamin B12 deficiency in apparently healthy elderly population visiting hospitals, as the results could help rationalize and direct the appropriate management of vitamin B12 status in elderly.

Methods : This was a time bound cross sectional study where apparently healthy individuals $>50$ years of age were interviewed, examined and investigated in order to study their vitamin B12 concentrations and associated clinical profile.

Results: A total of 103 participants were included. The mean vitamin B12 level of the study population was $327.07 \mathrm{pg} / \mathrm{ml}$ $(S . D=268.00)$. In the study, $43.7 \%$ ( $n=45$ patients) had low vitamin B12 levels (vitamin B12 deficiency) and $17.5 \%$ ( $n=18$ patients) had subnormal vitamin B12 levels. Among all the symptoms recorded neurological followed by cardiovascular symptoms were more commonly recorded in the patients with vitamin B12 deficiency. Distal dysesthesia was more commonly present in patients with deficient ( $p$ 0.044) than in patients with normal vitamin B12 levels. Ataxia and Romberg's sign positivity were more common in patients with vitamin B12 deficiency when compares to normal vitamin B12 levels ( $p$ 0.002 and 0.006 , respectively).

Conclusion: The study shows that vitamin B12 deficiency is a common problem among elderly and the treating physician should have high index of suspicion when they present with neurological complaints.

Keywords: Cobalamin deficiency, Neurological complaints, Subnormal vitamin B12 levels, Vitamin B12 deficiency

\section{MAIN TEXT}

Introduction

Vitamin B12 (Cobalamin) deficiency is common in elderly and various studies have suggested prevalence between 3 to $40 \% .^{(1,2,3)}$

Pernicious anemia though being a classical cause of vitamin B12 deficiency occurs only in a small subset of the elderly population. ${ }^{(4)}$

Food-cobalamin malabsorption, an inability to release cobalamin from food, is attributed mainly to atrophic gastritis and accompanying hypochlorhydria the prevalence of which increases with aging. ${ }^{(5,6)}$ However, studies reporting contradictory relationship between food-cobalamin malabsorption and aging exist and the actual relationship is yet to be established. (7)

As vitamin B12 is naturally found in animal products and vegetarianism is practiced more in India, the impact of insufficient intake (if any) of vitamin B12 in India is to be determined. ${ }^{(8,9)}$

The neuropsychiatric and hematological complications are potentially serious and necessitate investigations whenever a deficiency is suspected. However, the wide and non-specific spectrum of clinical presentations in patients poses a tough challenge to physicians suspecting vitamin B12 deficiency.(10)

Also, the need for an optimal management of mild deficiency or subnormal status of vitamin B12 levels is yet to be ascertained. ${ }^{(11)}$

\section{Methods}

This was a time bound cross sectional study where apparently healthy individuals $>50$ years of age visiting B J Medical College, Ahmedabad were interviewed, examined and investigated in order to study their vitamin B12 concentrations and associated clinical profile. 


\section{Eligibility criteria}

Individuals of $>50$ years of age randomly selected to represent apparently healthy elderly population.

\section{Exclusion criteria}

- Individuals $<50$ years of age

- Individuals with history of receiving vitamin b12 supplementation in the past one year

- Individuals who have undergone surgeries like total/partial gastrectomy and gastric bypass

- Individuals who have received radiotherapy

- Critically ill

- Diagnosed cases of

Pernicious anemia

Malabsorption syndromes

Chronic pancreatitis

Immunocompromised state

Hypothyroidism

Chronic liver diseases

\section{History and clinical examinations}

Information on symptoms was obtained by structured interview. A record of anemia symptoms, cardiovascular symptoms, respiratory symptoms, genito-urinary symptoms, gastrointestinal symptoms, and neurological symptoms were made.

A history of present and previous disease was obtained. In addition, current drug use, diet patterns, and addictions were also recorded.

All patients were then subjected to thorough general examination to look for clinical signs of cobalamin deficiency. The neurological examination comprised testing for higher mental function, vibration sense, joint position sense, cutaneous sensation, postural instability, hyporeflexia, muscular strength or presence of cerebellar signs.

\section{Investigations performed:}

a) Complete blood count

b) Serum vitamin B12 level

\section{Operational definition:}

B12 levels were then defined as low (or deficient) if $=187 \mathrm{pg} / \mathrm{ml}$ ), intermediate (or subnormal) if $188-299 \mathrm{pg} / \mathrm{ml}$ and normal if $=300 \mathrm{pg} / \mathrm{ml}$.

\section{Statistical method:}

The data were analyzed using IBM SPSS Statistics v23. Continuous variables were expressed as mean \pm standard deviation (SD). Different groups were compared using nonparametric test like 2 groups Mann-Whitney U test. To compare more than two groups Kruskal-wallis $\mathrm{H}$ test was used and if any significant difference was seen post-hoc analysis was done to study pairwise comparison. Jonckheere-Terpstra test was used for ordinal dependent variable and for other categorical data, Pearson `s Chi-square test was used. P value of $<0.05$ was considered significant.

\section{Results}

A total of 103 participants were included and comprised of 46 females and 57 males.

Mean age of the study population was 61.13 years ( $S . D=9.63$ ). Mean age of males and females in study population were 61.28 years $(S . D=10.00)$ and 60.94 years $(S . D=9.26)$ respectively.

The mean vitamin B12 level of the study population was $327.07 \mathrm{pg} / \mathrm{ml}(\mathrm{S} . \mathrm{D}=268.00)$. The mean vitamin B12 levels of females and males were $351.87 \mathrm{pg} / \mathrm{ml}(\mathrm{S} . \mathrm{D}=239.43)$ and $307.05 \mathrm{pg} / \mathrm{ml}$ (S.D=289.56) respectively. The distribution of serum vitamin B12 levels were same between various age groups defined in the study ( $p 0.970$ ) as well as between males and females ( $p$ 0.103).

In the study, $43.7 \%$ ( $n=45$ patients) had low vitamin B12 levels (vitamin B12 deficiency) and $17.5 \%$ ( $n=18$ patients) had subnormal vitamin B12 levels. A cumulative $61.2 \%$ ( $n=63$ patients) had either deficient or subnormal vitamin B12 levels.

No significant difference in prevalence of deficient or subnormal vitamin B12 levels were observed between various age groups ( $p 0.705$ ) or between genders ( $p 0.258$ ).

Among all the symptoms recorded neurological symptoms were more commonly recorded in the patients with vitamin B12 deficiency ( $p$ 0.005) or subnormal vitamin B12 levels ( $p$ 0.048) than in patients with normal vitamin B12 levels. In the study population, 67 patients reported some or the other neurological complaints. In them $55.2 \%$ ( $n=35$ patients) had vitamin B 12 deficiency and 20.9\% ( $n=14$ patients) had subnormal vitamin B12 levels. The rest $26.9 \%$ ( $n=18$ patients) had normal vitamin B12 levels.

Distal dysesthesia was the most common symptom recorded in $48.9 \%$ of vitamin B12 deficient ( $n=22$ patients) followed by generalized weakness in $31.1 \%$ of vitamin B12 deficient (14 patients). Distal dysesthesia was also the most common complaint in patients with vitamin B12 deficiency ( $p$ 0.044 ) or subnormal vitamin B12 levels ( $p$ 0.001) than in patients with normal vitamin B12 levels. Generalized weakness was more common in patients with vitamin B12 deficiency ( $p$ 0.008 ) than in patients with normal vitamin B12 levels. 
Cardiovascular symptoms were common in patients with vitamin B12 deficiency ( $p$ 0.003) than in patients with normal vitamin B12 levels. Gastrointestinal and other systemic complaints recorded when compared were not different between vitamin B12 deficient, subnormal or normal vitamin B12 level groups.

There was no significant difference in prevalence of hypertension, diabetes mellitus, chronic gastritis, coronary arterial events or cerebrovascular events between vitamin B12 deficient, subnormal or normal vitamin B12 level groups.

Mean serum vitamin B12 level in vegetarians was 291.20 $\mathrm{pg} / \mathrm{ml}(\mathrm{S} . \mathrm{D}=224.42)$ and in non-vegetarians it was $369.81 \mathrm{pg} /$ $\mathrm{ml}(\mathrm{S} . \mathrm{D}=308.35)$. However, no significant difference in prevalence of deficient or subnormal vitamin levels was found between vegetarians and non-vegetarians ( $p 0.394$ ).

People who admitted having addicting habits like drinking, betel nut chewing, smoking or other forms of tobacco use were not found to have increased prevalence of vitamin B12 deficiency or subnormal vitamin B12 status ( $p$ 0.088).

Pallor, hyperpigmented knuckles, or glossitis usually described as manifestations of vitamin B 12 deficiency were also found in patients with subnormal or normal vitamin B12 levels ( $p$ values of $0.790,0.525$, and 0.804 respectively).

Prevalence of decreased muscle strength (power), ataxia, impaired joint position sense, and Romberg's sign positivity were not same in patients with low, intermediate or normal vitamin B12 levels ( $p$ 0.009, 0.000, 0.038, 0.002 respectively). When compared pairwise, decreased muscle power and impaired joint position sense were more common in patients with vitamin B12 deficiency than in patients with normal vitamin B12 levels ( $p 0.017$ for muscle power and 0.035 for joint position sense). Ataxia and Romberg's sign positivity were more common in patients with vitamin B12 deficiency than in patients with subnormal ( 0.002 for ataxia and 0.015 for Romberg's sign) or normal vitamin B12 levels ( $p 0.002$ for ataxia and 0.006 for Romberg's sign).

No significant difference in presence of findings like paresthesia ( $p 0.127)$, postural instability ( $p 0.220)$, impaired vibration sense ( $p$ 0.153), hyporeflexia ( $p$ 0.491), Babinski`s

Table 2: Age-group wise and gender wise distribution of vitamin B12 levels

\begin{tabular}{|c|c|c|c|c|c|c|}
\hline \multirow{3}{*}{ Age (years) } & \multicolumn{6}{|c|}{ Gender } \\
\hline & \multicolumn{3}{|c|}{ Females $(n=46)$} & \multicolumn{3}{|c|}{ Males ( $n=57)$} \\
\hline & Low & Intermediate & Normal & Low & Intermediate & Normal \\
\hline $50-59$ & 9 & 4 & 10 & 12 & 6 & 7 \\
\hline $60-69$ & 3 & 2 & 7 & 11 & 2 & 6 \\
\hline 70-79 & 2 & 3 & 3 & 5 & 1 & 4 \\
\hline
\end{tabular}

sign ( $p$ 0.758), or cerebellar signs ( $p$ 0.368) were found between deficient, subnormal or normal vitamin B12 level groups.

$52.4 \%$ ( $n=54$ patients; 30 males, 24 females) of the study population were anemic (hemoglobin $=11.9 \mathrm{~g} / \mathrm{dl}$ ). Mean hemoglobin of the study population was $11.68 \mathrm{~g} / \mathrm{dl}$ (SD 2.75). Mean hemoglobin in men was $12.00 \mathrm{~g} / \mathrm{dl}$ (SD 3.07) and in women it was $11.29 \mathrm{~g} / \mathrm{dl}$ (SD 2.27).

Out of 30 males with anemia, 15 (50\%) had vitamin B12 deficiency, 1 (3.33\%) had subnormal vitamin B12 level and 14 (46.67\%) had normal vitamin B12 levels. Out of 24 females with anemia, 6 (25\%) had vitamin B12 deficiency, 6 (25\%) had subnormal vitamin B12 levels and 12 had normal vitamin B12 levels.

Mean hemoglobin of participants in vitamin B12 deficient group was $12.24 \mathrm{~g} / \mathrm{dl}$ (S.D=2.49). In participants with subnormal and normal vitamin B12 levels the mean hemoglobin was $12.33 \mathrm{~g} / \mathrm{dl}(\mathrm{S} . \mathrm{D}=3.05)$ and $10.76 \mathrm{~g} / \mathrm{d}$ $(S . D=2.70)$, respectively. The distribution of hemoglobin across various age groups defined in the study as well as their distribution between males and females were same ( $p$ values of 0.290 and 0.064 respectively). Also there was no difference in prevalence of anemia across various age groups ( $p 0.294$ ) or between genders ( $p$ 0.963). However, the mean hemoglobin levels and the prevalence of anemia were significantly different between participants with low and normal vitamin B12 levels (p 0.029 and 0.040 , respectively).

Tables and figures: To be placed along with results.

Table 1: Mean vitamin B12 levels across various age groups

\begin{tabular}{|c|c|c|}
\hline $\begin{array}{c}\text { Age group } \\
\text { (in years) }\end{array}$ & $\begin{array}{c}\text { Mean Vitamin } \\
\text { B12 }(\mathbf{p g} / \mathbf{m l})\end{array}$ & Std. Error \\
\hline $50-59$ & 321.063 & 36.3201 \\
\hline $60-69$ & 354.452 & 59.0369 \\
\hline $70-79$ & 285.722 & 40.6070 \\
\hline $80-89$ & 350.800 & 164.5471 \\
\hline
\end{tabular}


Darshana Makwana et al

www. pimr.org.in

\begin{tabular}{|l|l|l|l|l|l|l|}
\hline $80-89$ & 2 & 0 & 1 & 1 & 0 & 1 \\
\hline $90-99$ & 0 & 0 & 0 & 0 & 0 & 1 \\
\hline Total $(n=103)$ & $n=16$ & $n=9$ & $n=21$ & $n=29$ & $n=9$ & $n=19$ \\
\hline
\end{tabular}

Figure 1: Vitamin B12 status in study population

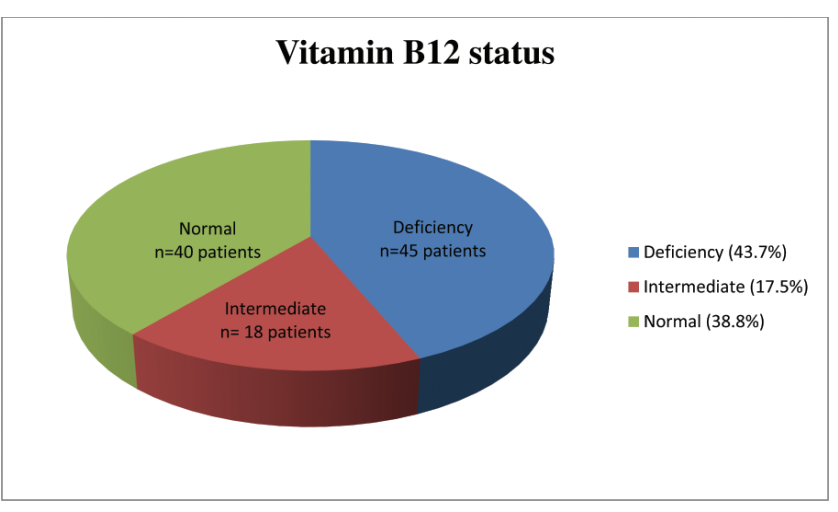

Figure 2: Clinical profile of patients with low, intermediate and normal vitamin B12 levels

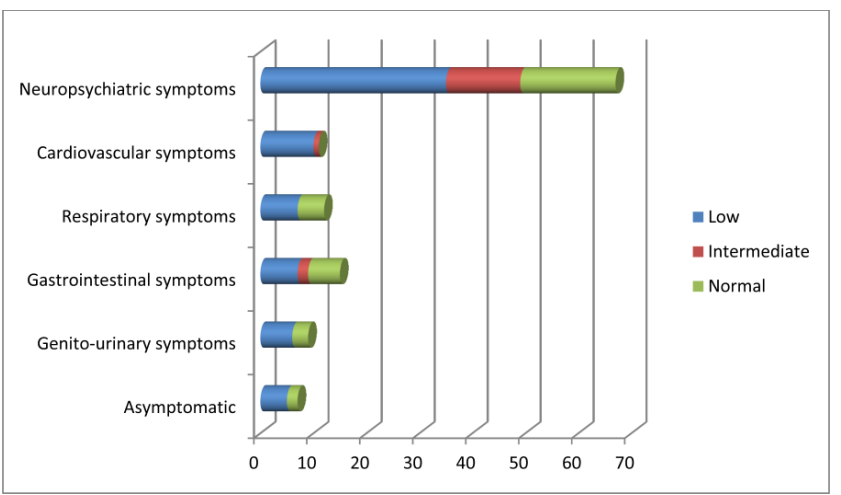

Note: Numbers along $\mathrm{X}$-axis indicates the number of participants in whom the complaints were recorded.

Figure 3: Clinical profile of patients with vitamin B12 deficiency $(n=45)$

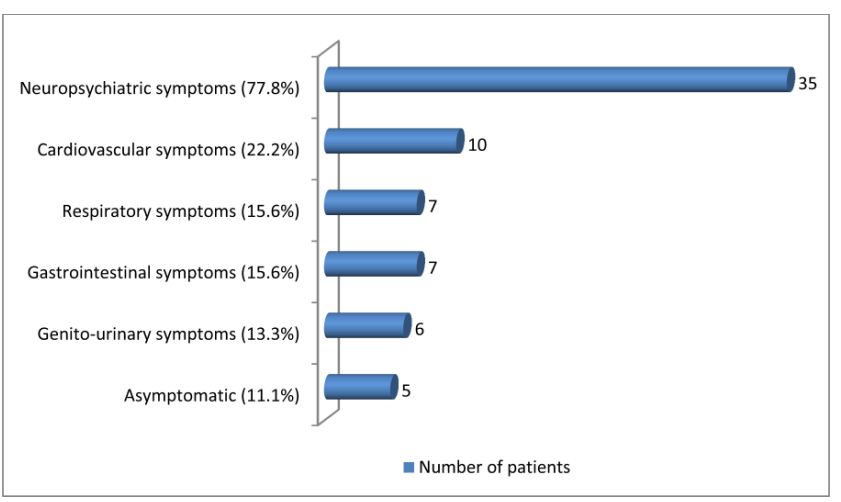

Figure 4: Distribution of hemoglobin between females and males

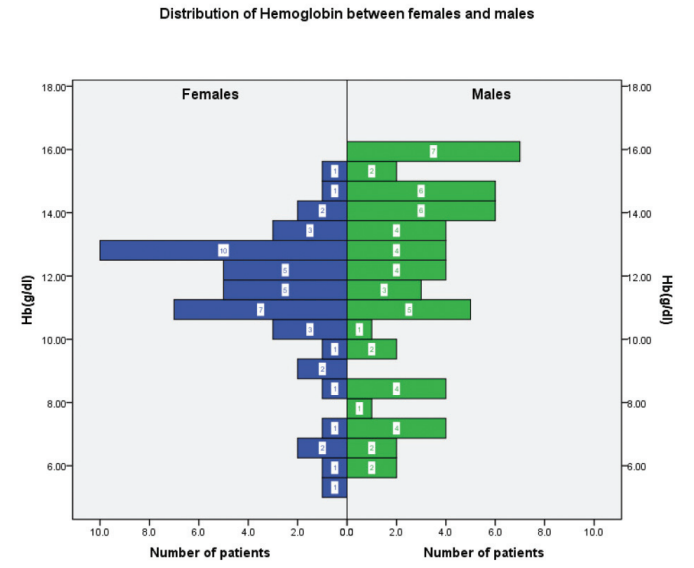

Figure 5: Distribution of hemoglobin across vitamin B12 status in females and males

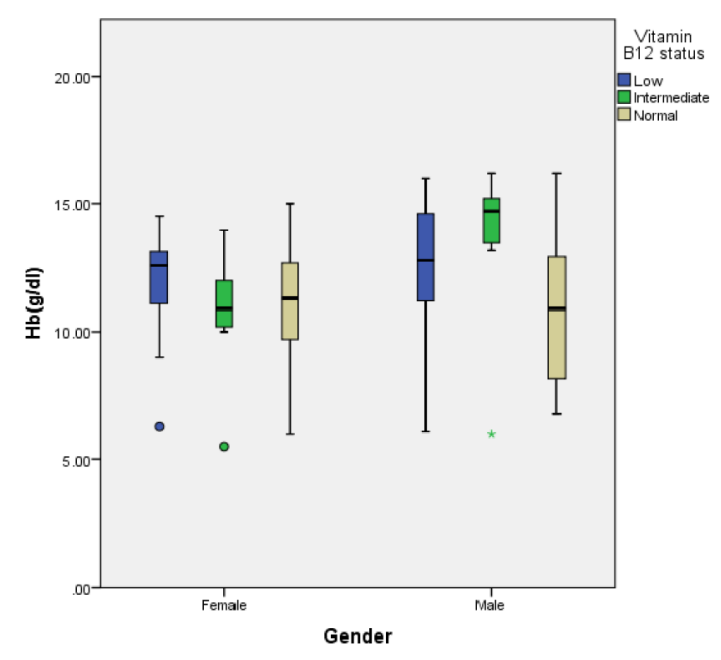

Discussion

The present study which included 103 participants with 57 males and 46 females showed that the prevalence of vitamin B12 deficiency was $43.7 \%$ (45 patients) in elderly aged above 50 years. These results were similar to study by Refsum et al which reported vitamin B12 deficiency in $47 \%$ of population aged between 27 and 55 years. On further tests $73 \%$ of the study population in Refsum et al would show metabolic signs of vitamin B12 deficiency. ${ }^{(12)}$ In the present study, 63 patients $(61.2 \%)$ had vitamin B12 levels considered deficient or subnormal. 
Fernandes-Costa et al (1985) had previously reported significantly higher serum B 12 values in females than in males with mean serum $B 12$ values of 819 and $647 \mathrm{pg} / \mathrm{mL}$ in females and males. ${ }^{(13)}$ Other investigators have reported similar findings. ${ }^{(14,15)}$ However studies that have found no difference in mean B 12 values between both sexes also exist. ${ }^{(16)}$ In the present study too the mean vitamin B12 levels were greater in females than in males with the mean vitamin B12 level of females and males being $351.87 \mathrm{pg} / \mathrm{ml}$ (S.D=239.43) and $307.05 \mathrm{pg} / \mathrm{ml}(\mathrm{S} . \mathrm{D}=289.56)$ respectively and was not significantly different. Taken together, the study does not provide sufficient evidence on which to predict a difference in B 12 status by gender.

Though some studies in the past have reported age related decline in vitamin B12 levels owing to increasing prevalence of atrophic gastritis in elderly contradictory studies also do exist. ${ }^{(16,17,18,19)}$ In the present study, vitamin B12 doesn't seem to significantly decrease with aging suggesting that aging alone may not be the risk factor for developing vitamin B12 deficiency.

Neurological system was the most commonly involved system with distal dysesthesia followed by generalized weakness being the most common complaints. Neurological complaints were documented in $77.8 \%$ of patients with vitamin B12 deficiency (35 out of 45 ) and the same percentage of people with subnormal vitamin B12 levels (14 out of 18) which was similar to the results observed by Healton et al (1991) and Savage et al (1994) who reported neurological complications in $75-90 \%$ of their study population. $(20,21)$ $29.55 \%$ ( $n=13$ out of 44 ) of the patients with distal dysesthesia and $20 \%$ ( $n=4$ out of 20 ) of the patients with generalized weakness in the study population had subnormal vitamin B12 levels and not frank deficiency implying that treatment should be considered even in patients with subnormal vitamin B12 levels.

Though cardiovascular symptoms like chest discomfort and palpitations were reported more often in patients with vitamin B12 deficiency, majority of those who reported these complaints also had anemia which could have interfered with the results.

Studies have shown B12 deficiency as an independent risk factor for cerebrovascular and coronary events. ${ }^{(22,23)}$ However, in the present study, such association of vitamin B12 deficiency was not seen with coronary artery events or cerebrovascular events. This discrepancy could be attributed to small study sample.

In the study both vegetarians and non-vegetarians were equally prone to develop vitamin B12 deficiency which contradicts other studies which reported increased deficiency in vegetarians. ${ }^{(24,25,26)}$ This finding possibly indicates that nonvegetarians in India are only "occasional" meat eaters.
Smoking, alcohol and tobacco use are known risk factors for deficiency of many vitamins including vitamin B12. ${ }^{(8,27,28)}$ In the present study, people who admitted having addicting habits were not found to have increased prevalence of vitamin B12 deficiency or subnormal vitamin B12 status. However, strong inferences were not made as the details regarding the type or severity of addicting habits were not collected. It will be interesting to see if any association between various addicting habits and vitamin B12 deficiency will be found in future studies.

Pallor, hyperpigmented knuckles, or glossitis are usually described as manifestations of vitamin B 12 deficiency. ${ }^{(29)}$ These features were also present in non-deficient population and cannot be considered as reliable indicators.

Neurological manifestations of vitamin B12 deficiency include sensory disturbances in the extremities, impairment of vibratory and position sense, motor disturbances, cognitive changes, visual disturbances, insomnia, impotency, and impaired bowel and bladder control. ${ }^{(8,30,31)}$ In the study, neurological findings like paresthesia, postural instability, impaired vibration sense, hyporeflexia, Babinski’s sign, or cerebellar signs were also found in population with subnormal or normal vitamin B12 levels. Therefore these cannot be considered as credible indicators of vitamin B12 deficiency, at least in elderly.

Decreased muscle power and impaired joint position sense were more common in patients with vitamin B12 deficiency when compared with the normal participants. However their prevalence was same when it was compared with participants with subnormal vitamin B12 levels ( $p 1.00$ for decreased power, p 1.000 for impaired joint position sense). Therefore their presence may indicate deficient or subnormal vitamin B12 status.

Ataxia and Romberg's sign positivity were seen commonly in patients with vitamin B12 deficiency than in patients with subnormal or normal vitamin B12 levels and could be more specific indicators of vitamin B12 deficiency.

Thus the present study suggests that most of the patients could be developing neurological manifestation much before they develop overt vitamin B12 deficiency, once again, reinforcing the thought of considering treatment of patients with subnormal vitamin B12 levels, though not agreed by everyone. ${ }^{(11)}$

In the present study, which consisted of participants above 50 years of age, aging or gender did not influence the distribution of hemoglobin or prevalence of anemia (hemoglobin $=11.9 \mathrm{~g} / \mathrm{dl}$ ). Surprisingly when hemoglobin distribution was studied against vitamin B12 levels of the population it was found that the population with normal vitamin B12 levels had lower mean hemoglobin levels when compared to patients with deficient or subnormal vitamin B12 
status, indicating that there were other causes of anemia which have to be considered while evaluating anemia in the elderly population with anemia of chronic disease and iron deficiency probably still being the common culprits. ${ }^{(32,33)}$

\section{Conclusion}

The present study shows that vitamin B12 deficiency is a common problem among elderly and the treating physician should have high index of suspicion when elderly patients present with neurological complaints like distal dysesthesia and weakness. Detailed neurological examination should be performed even in asymptomatic elderly patients and if findings consistent with vitamin B12 deficiency are found treatment should be considered, even when vitamin B12 levels are found to be subnormal. Anemia, glossitis etc. are classical but not the common features of vitamin B12 deficiency and cannot be considered as reliable indicators of vitamin B12 deficiency. Future prospective and interventional studies with larger population and vitamin B12 supplementation will help in proposing stronger recommendations with respect to treatment of patients with subnormal vitamin B12 levels.

\section{Limitations}

- This study conducted in apparently healthy population visiting health care need not be necessarily healthy and may not be representative of general elderly population.

- Small study population

- Absence of structured tool to assess dietary intake of vitamin B12 or details regarding addicting habits.

\section{REFERENCES}

1. Andres E, Loukili NH, Noel E, Kaltenbach G, Abdelgheni $M B$, Perrin AE, et al. Vitamin B12 (cobalamin) deficiency in elderly patients. CMAJ 2004; 171: 251-9.

2. Pautas E, Cherin P, De Jaeger C, Godeau P. Vitamin B 12 deficiency in the aged. Presse

Med1999; 28:1767-70.

3. van Asselt DZ, Blom HJ, Zuiderent R, Wevers RA, Jakobs $C$, van den Broek WJ, et al. Clinical significance of low cobalamin levels in older hospital patients. Neth J Med 2000;57:41-9.

4. Carmel R. Current concepts in cobalamin deficiency. Annu Rev Med 2000;51:357-75.

5. Andres E, Affenberger S, Vinzio S, Kurtz JE, Noel E, Kaltenbach $G$, et al. Food-cobalamin malabsorption in elderly patients: clinical manifestations and treatment. Am J Med 2005;118: 1154-9.
6. Andres E, Noel E, Kaltenbach G, Perrin AE, Vinzio $S$, Goichot B, et al. Vitamin B12 deficiency with normal Schilling test or non-dissociation of vitamin B12 and its carrier proteins in elderly patients. A study of 60 patients. Rev Med Interne 2003; 24:218-23.

7. van Asselt DZ, van den Broek WJ, Lamers CB, Corstens $\mathrm{FH}$, Hoefnagels WH. Free and protein-bound cobalamin absorption in healthy middle-aged and older subjects. J Am Geriatr Soc.1996; 44: 949-953.

8. Dietary Reference Intakes: Thiamin, Riboflavin, Niacin, Vitamin B 6, Folate, Vitamin B 12 , Pantothenic Acid, Biotin, and Choline. Washington, DC: National Academy of Sciences; 1998. Panel on Folate, Other B Vitamins, and Choline; Institute of Medicine. Vitamin B 12. pp. 306-356.

9. Edelstein, Sari. India has more vegetarians than everywhere else in the world combined. Food Science, An Ecological Approach 2013. Jones \& Bartlett Publishers. Pp. Page 281. ISBN 978-1-4496-0344-1

10. Reynolds E. Vitamin B12, folic acid, and the nervous system. Lancet Neurol 2006; 5: 949-60.

11. Carmel R. How I treat cobalamin (vitamin B12) deficiency. Blood.2008: 112; 2214-21.

12. Refsum M, Yajnik CS, Gadkari $M$, et al. Hyperhomocysteinemia and elevated methylmalonic acid indicate a high prevalence of cobalamin deficiency in Asian Indians.

Am J Clin Nutr 2001;74:233-41.

13. Fernandes-Costa F, van Tonder S, Metz J. A sex difference in serum cobalamin and transcobalamin levels. Am J Clin Nutr. 1985;41: 784-786.

14. Gender and age influence blood folate, vitamin B12, vitamin B6, and homocysteine levels in European adolescents: the Helena study. Gonzalez-Gross M, et al. Nutr Res. 2012.

15. Plasma total homocysteine, folate, and vitamin B12 status in Korean adults. Lim HS, et al. J Nutr Sci Vitaminol (Tokyo). 2002.

16. Vitamin B12 levels and age. Hitzhusen JC et al. Am J Clin Pathol. 1986.

17. Krasinski SD, Russell RM, Samloff IM, Jacob RA, Dallal GE, McGandy RB, Hartz SC. Fundic atrophic gastritis in an elderly population: Effect on hemoglobin and several serum nutritional indicators. J Am Geriatr Soc. 1986; 34 :800-806. 
18. Scarlett JD, Read H, O'Dea K. Protein-bound cobalamin absorption declines in the elderly. Am J Hematol. 1992;39:79-83.

19. Ralph Carmel, Ralph Green, Donald W Jacobsen, Karsten Rasmussen, Mircea Florea, and Colleen Azen. Serum cobalamin, homocysteine, and methyl malonic acid concentrations in a multiethnic elderly population: ethnic and sex differences in cobalamin and metabolite abnormalities. Am J Clin Nutr 1999.

20. Healton EB, Savage DG, Brust JC, Garrett TJ, Lindenbaum J. Neurologic aspects of cobalamin deficiency. Medicine (Baltimore) 1991;70:229-245.

21. Savage D, Gangaidzo I, Lindenbaum J. Vitamin B 12 deficiency is the primary cause of megaloblastic anemia in Zimbabwe. Br J Haematol. 1994;86: 844-850.

22. Homocysteine and the Risk of ischemic stroke in a Triethnic cohort : The Northern Manhattan Study. Ralph L. Sacco et al. Stroke. 2004; 35; 2263-2269.

23. Paul Ganguly and Sreyoshi Fatima Alam. Role of homocysteine in the development of cardiovascular disease. Nutr J. 2015; 14:6.

24. Herrmann W, Schorr H, Obeid R, Geisel J. Vitamin B12 status, particularly holotranscobalamin II and methylmalonic acid concentrations, and hyperhomocysteinemia in vegetarians. Am J Clin Nutr 2003; 78: 131-6.

25. Fiona O'Leary and Samir Samman. Vitamin B12 in health and disease. Nutrients. 2010 Mar; 2(3): 299-316.

26. Carol L Zeuschner et al. Vitamin B12 and vegetarian diets. Med J Aust 2013; 199(4 Suppl): S27-S32.

27. Alberto Fragasso et al. Functional vitamin B12 deficiency in alcoholics: An intriguing finding in a retrospective study of megaloblastic anemic patients. Eu J Int Med. 2010.

28. Alberto Fragasso et al. Holotranscobalamin is a useful marker of vitamin B12 deficiency in alcoholics. ScientificWorldJournal. 2012; 2012: 128182.

29. Aaron $S$ et al. Clinical and laboratory features and response to treatment in patients presenting with vitamin B12 deficiency- related neurological syndromes. Neurol India. 2005;53(1):55-8.

30. Dali-Youcef N, Andrès EAn update on cobalamin deficiency in adults. QJM. 102:17,.2009.
31. Carmel R, Melnyk S, James SJ. Cobalamin deficiency with and without neurologic abnormalities: differences in homocysteine and methionine metabolism. Blood. 2003;101: 3302-3308.

32. Anemia in the elderly. DOUGLAS L. SMITH, M.D.,University of Wisconsin Medical School, Madison, Wisconsin. Am Fam Physician. 2000 Oct 1; 62(7):1565-1572.

33. Prevalence of anemia in persons 65 years and older in the United States: evidence for a high rate of unexplained anemia. Jack M Guralnik, Richard S. Eisenstaedt, Luigi Ferrucci, Harvey G. Klein and Richard C. Woodman. Blood 2004 104: 2263-2268; https://doi.org/10.1182/blood2004-05-1812.

How to cite this article : Makwana D, Deepak Kumar $P$, Wadkar P Vitamin B12 concentrations and clinical spectrum of apparently healthy elderly population. Perspectives in Medical Research 2020; 8(1):48-54

Sources of Support: Nil, Conflict of interest: None declared 